\title{
Application of cognitive-behavioral therapy techniques as the method of early intervention in the therapy of psychotic disorders
}

\author{
Oleksandr Avramchuk', Khrystyna Shalak ${ }^{2}$ \\ 1 Department of Clinical Psychology, Ukrainian Catholic University, Lviv, Ukraine \\ 2 Lviv Regional Psychiatric Hospital "Zaklad", Lviv, Ukraine
}

\begin{abstract}
Psychotic disorders occupy an essential place in the structure of general morbidity of the population, affect quality and life expectancy, reduce working capacity and often cause disability. Psychotic symptoms cause significant distress in patients and lead to alterations in important spheres of life, such as social functioning, and could be the cause of suicidal or socially dangerous behavior. According to the World Health Organization, schizophrenia affects about 21 million people, worldwide. The onset of the disease occurs in adolescents and early adulthood, with a range of $15-29$ years.

The present paper aims to investigate the features of the use of cognitive-behavioral therapy techniques and their effectiveness in the treatment of early stages of psychosis. This study presents already existing systemic literature reviews and meta-analyses on the topic of cognitive-behavioral therapy (CBT) of psychotic disorders, namely devoted to CBT of the first psychotic episode. Besides, it examines the effectiveness of the use of CBT not only in the prodromal stage of psychosis and during its manifestation, as well as in the remission period as a method for preventing recurrence.

According to the data, CBT is a useful and scientifically based method in the combined therapy of patients in the early stages of the course of psychotic illness. CBT early interventions reduce positive and negative symptoms severity, improve access to engagement with treatment, which also reduces hospital admission and relapse rates.
\end{abstract}

Keywords: Mental health, psychiatry, psychology, psychosis, schizophrenia 


\section{HIGHLIGHTS / KEY POINTS}

- Discussion of early interventions for the treatment of first psychotic episode.

- Complex early intervention based on CBT influence on positive and negative symptoms.

- Complex early intervention based on CBT influence on social functioning and relapse prevention.

- Standardized CBT protocols for depression or anxiety disorders may be used for the treatment of comorbidities for patients with psychotic disorders.

\section{INTRODUCTION}

ccording to the National Institute of Mental Health, in 2015, in the USA around 100,000 people had the first psychotic episode. Three in every 100 people have at least one psychotic episode in their lives (National Institute of Mental Health 2015).

According to the World Health Organization, schizophrenia affects about 21 million people all over the world. The onset of the disorder occurs between 15-29 years, adolescents and early adulthood (World Health Organization 2012). People who have 2.5 times more often psychotic disorders, could die in young age due to somatic causes (cardiovascular, metabolic and infectious disorders).

According to the official statistics of the Ministry of Health of Ukraine, in 2015, the incidence of psychotic disorders (schizophrenia, schizotypal and delusion disorder) was 15.82 per 100,000 population. The prevalence of schizophrenia was 292.6 per 100,000 population (Ministry of Health of Ukraine 2016).

Psychotic disorders occupy an essential place in the structure of the general morbidity of the population, affect quality of life and life expectancy, reduce working capacity and often cause disability. Psychotic symptoms cause significant distress, lead to violations in important spheres of life and social functioning. They also could be the cause of the suicidal or socially dangerous behavior and affective disorders. Therefore, the issues of effective treatment and prevention of these disorders, as well as social rehabilitation of patients, are relevant.

According to the recommendations of the National Institute for Health and Care Excellence (NICE 2014) and the World Health Organization (WHO 2012) for the treatment of psychosis and schizophrenia in adults, cognitive-behavioral therapy (CBT) is the method of choice among all psychotherapeutic interventions for patients with the early or first-episode psychosis (in combination with second-generation antipsychotics). It is recommended to initiate at the end of the acute phase or during it in inpatient settings.

In the clinical protocol by the Ministry of Health of
Ukraine is noted that treatment of the primary psychotic episode must be both biological and psychosocial (Ministry of Health of Ukraine 2007). Psychosocial intervention, psycho-educational work with the patient's family, cognitive-behavioral, and other forms of psychotherapy (not included) are recommended. The primary aim for this group of patients is to help them to understand their disorder, the essence of the therapeutic process, and to achieve the recovery of social functioning (Ministry of Health of Ukraine 2007). However, the leading and often the only approach in psychosis treatment in Ukraine is biomedical (the use of medical treatment). In our country, we have no protocols or manuals for conducting cognitive-behavioral therapy for patients with early or first-episode of psychosis. In our opinion, it is one of the points why psychotherapeutic and psychological interventions are rarely used and are non-mandatory.

The purpose of our literature review is to explore the features and evidence-based data on the use of CBT techniques and their effectiveness in treatment of psychosis in the early stages. This study complements already existing systematic reviews and meta-analyses on the topic of cognitive-behavioral therapy of psychotic disorders, in particular, those applied in the complex treatment of the first psychotic episode. This review contains an analysis of newer articles on this topic, as well as those that were not included in previous studies. Also, it reviewed CBT as a method for preventing relapses.

With the help of this study, it is possible to form the recommendations for the implementation of cognitivebehavioral therapy for the first psychotic episode as a method of early psychotherapeutic intervention in the complex treatment.

\section{METHODS}

Previous searches related to the topic of the article were done in PubMed, Medline, Web-of-Science, BIOSIS, Cochrane library and Research Gate. Two studies were found: "Early intervention services, cognitive-behavioral 


\section{SYSTEMATIC REVIEW}

therapy and family intervention in early psychosis: a systematic review" (Bird \& Premkumar 2010) and "Cognitive Behavioral Therapy in Prodromal Psychosis" (Addington et al 2012). It confirmed the usefulness of a new report, which would include a more recent study on the topic.

For our review, we used the following inclusion criteria:

n The research had to be published no more than 10 years ago.

w The research had to be related to the use of cognitive-behavioral therapy for treatment of patients with psychotic disorders in the early stages. These include prodromal phase (high risk of developing psychosis), first psychotic episode, recovery and remission after the first psychotic episode.

n The articles had to be published in authoritative scientific research resources: PubMed, Medline, Webof-Science, BIOSIS, Cochrane library and Research Gate.

Meta-analyses and system reviews, studies in which patients had a psychotic episode before or were constantly ill, studies involving patients under 16 years of age were excluded from the study.

The search was carried out using the following keywords: "CBT" and "Early psychoses," "CBT" and "First psychotic episode," "CBT" and "Schizophrenia onset," "CBT for psychoses," "Psychotherapy for psychoses." We obtained 135 articles. Due to the screening of duplicate items and selection according to inclusion and exclusion criteria, 17 studies were selected for further analysis 111 randomized controlled trials and 6 case studies).

For our studies, we reviewed the following aspects: 1) the purpose, the main tasks and the hypothesis of the study, 2) research method, 3) study participants, inclusion criteria and exclusions for which they were selected, 4) how the study was conducted (interventions, duration, whether there was a control group, monitoring, scales, and questionnaires were used for this), 5) the primary results of the study: was the intervention effective, did the mental state of the participants change.

We analyzed the weaknesses and controversial points of each study. Also we reviewed the information about CBT protocols, manuals, recommendations and techniques that were used.

\section{RESULTS AND DISCUSSION}

\section{Generalized analysis of CBT for psychosis}

From the duration of the untreated primary psychosis of people with schizophrenia often depends on its prognosis (Bird \& Premkumar 2010). According to the NICE protocol, CBT and family therapy are recommended among psychotherapeutic interventions for schizophrenia and the first psychotic episode (NICE 2014). The CBT should last at least 16 sessions and aims to educate patients to see the connections between their thoughts, feelings, actions, and symptoms. During intervention analyzing the peculiarities of perception and beliefs of patients, they learn to monitor thoughts, feelings also behavior independently. Developing the alternative collaborative ways of coping with disorders symptoms reduce distress and improve functioning (NICE 2014).

Therapy aims to create settings where a person will not feel like a passive therapeutic object. Such an attitude helps to awaken a more functional sense of self. Open disclosure of information to a person regarding her condition and encouragement for joint decisionmaking are welcomed.

The goals of the CBT for psychosis include interventions aimed not only at the symptoms of the disease, but also their outcomes: isolation from family and friends, depression, increased risk of self-harm, aggression, substance abuse, etc. The aim of therapy as early intervention is to increase understanding of the psychotic disorder, improve adaptation to it, boost self-esteem, reduce emotional distress, management comorbid states and prevent relapses. That is especially important in the critical period of 3-5 years after the first episode (Bird \& Premkumar 2010). The CBT aims to reduce the distress caused by delusions and hallucinations, also to teach a person the strategies for managing residual symptoms (Gleeson \& McGorry 2003).

The CBT approach focuses on the person as a personality, and work is underway to realize their potential and their limitations, the development of a costrategy, and the construction of realistic plans for the future (Addington 2005).

\section{A generalized analysis of previous studies and presentation of the data}

There are some clinical trials of CBT for psychotic disorders, and most of them indicate its effectiveness. A systematic review and meta-analysis of 2010 compared the efficacy of early intervention services, cognitivebehavioral therapy and family intervention (Bird \& Premkumar 2010). Applying early intervention services and family interventions showed to reduce the severity of the symptoms, the rate of relapse and hospitalization, while CBT has reduced the severity of the signs and had little effect on relapse and hospitalization. In 2011 a systematic review of studies on the effectiveness of various interventions at early stages of development of psychotic disorders, included the prodromal phase 
and the first psychotic episode (Marshall 2011). It also confirmed the efficacy of combined risperidone and CBT.

In 2012, a survey of 20 randomized trials was conducted to compare the efficacy of CBT and other psychosocial interventions in schizophrenia (Jones \& Hacker 2012). In this study, CBT compared with family therapy, counseling, psychoeducation, group therapy and relaxation therapy. The analysis of the research did not show a significant difference between the effectiveness of these interventions, but the CBT showed a longer positive effect on distress, emotional regulation, and depression in psychotic patients.

In 2013, a meta-analysis carried out on the effectiveness of psychological, pharmacological and nutrition interventions in the prevention of psychosis in high-risk patients (early interventions to prevent psychosis: systematic review and meta-analysis). In this study 11 studies were included. The average positive effect was shown by $\mathrm{CBT}_{\text {; }}$ very low efficiency omega-3 fatty acids.

In 2014, a meta-analysis of 48 studies on the effectiveness of psychological interventions in patients with psychosis compared six types of psychosocial interventions (Turner \& van der Gaag 2014). It found that CBT is more effective in the treatment of positive symptoms, and social skills training is more effective in reducing of negative symptoms. Other interventions were less effective: befriending, cognitive recovery, psychoeducation, counseling based on Carl Rogers' client-centered therapy.

The Nowak \& Sa Sabariego systematic review (2016) included 50 studies and aimed to determine the effect of CBT on the incapacity of patients with schizophrenia and the recovery process, showed a positive effect of CBT on negative symptoms and social functioning.

Summarizing the articles, we determined the impact on the positive and negative symptoms, the effect on the comorbid states and symptoms of depression, anxiety and low self-esteem, changes in social functioning, the effect on relapse prevention.

According to the obtained data, the inclusion criteria in the study were an average age of 18-25 years and the presence of current or fluctuation of psychotic symptoms of moderate/severe degree with the onset of psychotic disorder over the past two years. Psychotic symptoms fell under the criteria of DSM-IV and ICD10 for schizophrenia, schizotypal disorder, delusion disorder, or other unspecified psychosis. Individual studies also included people with high-risk psychosis criteria according to the questionnaires "Comprehensive Assessment of At-Risk Mental States" (CAARMS) (Morrison 2012, Phillips et al 2012) and At-Risk Mental
State (ARMS) (van der Gaag et al 2012). In most cases, an additional inclusion criterion has been treatment with at least one atypical antipsychotic without changing medication for at least the last two months (Lecomte et al 2009, Edwards et al 2011, Gleeson et al 2011, Phillips et al 2012, Chung et al 2012, Singer et al 2013, Bechdolf et al 2017). Preference was given to patients with psychotic disorders without an affective component.

Typical exclusion criteria are organic brain and neurological disorders, abuse on alcohol or drugs, pregnancy and lactation, antidepressant therapy, mood stabilizers and ECT, inability to provide informed consent and intellectual disability. Some studies have considered the previous experience of CBT, social training or other psychosocial interventions at the time of the study (Jackson et al 2008, Lecomte et al 2009, Singer et al 2013).

\section{Influence of CBT on positive and negative symptoms}

A previous meta-analysis of the efficacy studies of the CBT for delusions by Mehl and Werner (2015) point to a moderate positive effect of psychotherapy in comparison with only medication treatment. At the same time, the analysis indicates to the slight superiority of the CBT-delusions over other psychological interventions. A randomized controlled study by Daniel Freeman (2015) showed a significant reduction in anxiety and delusional ideas, which confirmed the efficacy of CBT in patients with persecutory delusions.

In the context of cognitive-behavioral analysis, hallucinations ("voices") are considered as pathogens that cause certain cognitive processes. The patient thinks about them, evaluates and interprets that in the future causes an emotional reaction. The goal of CBT is to decrease the negative effects of "voices" on the patient's life by changing their attitude. The goal is to prevent the patient from maintaining them, checking if they can harm or control his actions.

The "voices" examination is analogous to the analysis of complaining. The patient learns to observe in what situations they appear, what is their content, how often they arise,, and, finally, what emotions and behavioral reactions they cause. The patient records the appearance of hallucinations (if necessary in writing or on a voice recorder). The point is that not the "voices" themselves cause stress, but the emotions and thoughts associated with them. Therefore, the task of the therapist is to work with the patient to test his assessment of "voices." The patient learns to respond to them more calmly and for this purpose behavioral experiments. During them, the patient specifically acts contrary to the "voices" and checks whether they are as omnipotent as he thought. 


\section{SYSTEMATIC REVIEW}

In a study by Tania Lecomte and colleagues ( $n=129$, age 18-35), CBT interventions were aimed at testing cognitive hypotheses of delusion content or content of hallucinations and finding alternatives (Lecomte et al 2009). At the same time, the therapy sessions covered stress, alcohol and drug use. An essential aspect of the approach used during the research was the formation of effective co-strategies, the achievement of personal goals, the reduction of distress, the search for individual ways of support. The following techniques of CBT, such as normalization of experiences, using Socratic questions, testing of thoughts, STEB-analysis, were used. They did not address directly at the symptoms of the disorder, but there applied was a decrease in the positive and negative symptoms. The CBT group showed better results on the signs and increased selfesteem compared to the control group (Lecomte et al 2009).

In literary sources, the system of early interventions in the primary psychotic episode appears as a multidisciplinary set of activities that include both pharmacotherapy and cognitive-behavioral interventions. The results of the study on the effectiveness of clozapine and cognitive-behavioral therapy in patients with the primary psychotic episode demonstrated a reduction in the intensity of symptoms and prompted a faster response to drug treatment when used in combination with CBT (Edwards et al 2011). The Systematic Treatment of Persistent Psychosis (STOPP) program, which used in that trial, included CBT intervention: "A Psychological Approach to Facilitating Recovery in Young People with First-Episode Psychosis" (Herrmann-Doig 2002). Sessions were twice a week for 12 weeks, minimum length of 15 sessions. At the 12th week, all groups showed improvement. The authors of the study indicated that the independent application of CBT can be considered as an alternative to patients with a low risk of developing psychosis or those who refuse antipsychotic therapy.

The study of Lisa J. Phillips and colleagues also investigated the influence of CBT interventions with antipsychotics. After 12 months of therapy, the proportion of developing the psychosis in the groups followed: CBT and placebo - 9.6\%; CBT and risperidone - $10.7 \%$; supporting counseling and placebo - $21.8 \%$ (Phillips et al 2012). However, the authors of the study indicate a lack of significant statistical difference $(p=$ 0.60 ). In all three groups demonstrated the same level of improvement in function and reduction of negative symptoms.

It should be noted that schizophrenia is associated with neurocognitive problems of varying degrees, which have an impact on social functioning. The main negative symptoms of schizophrenia include abulia, a decrease in the motivational function, anhedonia and emotionalaffective flattening. Behavioral techniques of CBT are considered as the primary therapy in the treatment of these symptoms. Among them self-monitoring behavior, activity recording and activity scheduling, rating activities of the most satisfying activities, and social skills training (Morrison 2009).

In following, studies included the patients with the high risk or ultrahigh risk for developing psychosis. The risk for psychosis typically appeared in those who had a close family member with psychosis (genetic factor). By conducting a preliminary review in most metaanalyses and reviews, these groups were included in the exclusion groups. When we were choosing them for evaluation, we wanted to assess the different opportunities and perspectives of the use of clinical trials for clinical practice, including for people at risk for later developing an imminent psychosis.

The six months of routine treatment as usual and Cognitive Behavioral Therapy for Subjects at Ultrahigh Risk for Developing Psychosis (CBTuhr) showed better results in reducing subclinical psychotic symptoms in patients with a high risk of developing psychosis (van der Gaag et al 2012). The CBT interventions in this study based on Paul French and Anthony P. Morrison recommendations (Early Detection and Cognitive Therapy for People at high risk of developing psychosis: A Treatment Approach, 2004), which also added to psychoeducation for the role of dopamine and cognitive disturbances. This group went to 26 sessions of CBT, and as results, ten patients developed psychosis. In the TAU group, psychosis has come from 22 participants.

An experimental group in the study of Bechdolf et al (2017) conducted "The Cognitive Behavior Therapy for People at Risk of Psychosis manual. A Treatment Manual" (Bechdolf et al 2010). As a result, there was a clinically significant difference between the development of the psychotic episode in both groups, but there was no statistical difference between them. CBT showed a significantly better outcome and a lower dropout in the study than clinical management and placebo and clinical management and antipsychotic $(p<0.05)$. These data may indicate better compliance in the experimental group. The CBT has had 30 individual 50-minute sessions for 12 months. Therapy included building formulation, homework, and psychoeducation about positive and negative symptoms. Patients were trained in self-monitoring of symptoms and provocative thoughts, normalization of psychotic experience, the search for alternative explanations and their verification by behavioral experiments. The training of social skills was included to prevent relapse. 


\section{SYSTEMATIC REVIEW}

The results of two independent studies by Anthony $P$. Morrison and colleagues demonstrated the efficiency of integrated implementation of CBT for treatment of early psychoses. The incidence of psychosis was approximately the same in two groups in the 2012 study, one of them using the CBT, other only monitoring the mental state (Morrison, 2012). However, the severity of the occurrence of psychotic episodes significantly reduced in the group where cognitive therapy was conducted. In 2018 the same author and colleagues compared the treatment of antipsychotics with independent using CBT and in the complex (Morrison et al 2018), and the Positive and Negative Symptom Scale (PANSS) score significantly decreased in the combined use of antipsychotics and CBT.

In the 2012 study, cognitive therapy was held once a week, with a total treatment duration of up to 26 weeks, plus up to four booster sessions in the next six months. The state of the participants observed after the intervention in 12 to 24 months. Cognitive therapy included an assessment of the mental state, the building of the formulation, the normalization of psychotic experience, and behavioral experiments. For CBT interventions used "Cognitive-behavioral therapy for individuals at high risk of developing psychosis" by Addington \& Mancuso (2009). While interventions in the CBT in the 2018 study consisted of 26 sessions based on protocol "A manualised treatment protocol to guide delivery of evidence-based cognitive therapy for people with distressing psychosis: learning from clinical trials" (Morrison 2016). We understand that the results of both studies cannot be interpreted as typical, but they can complement each other by pointing out the merits and importance of an integrated approach.

Analyzing the studies as mentioned earlier it is should be noted that the use of CBT-based interventions has a positive effect on positive and negative symptoms, but most studies did not show a statistically significant difference between the effectiveness of psychotherapy and drug treatment. At the same time, the use of CBT in complex interventions contributed to the formation of better compliance, reduced psychotic symptoms and the duration of pharmacological treatment.

Influence on social functioning and relapse prevention

The ability to detect early signs of psychosis is essential in preventing of a relapse and is a part of the CBT plan of psychosis. Studies indicate a feeling of fear, reduced mood, helplessness, hopelessness, shame or anxiety usually precedes a relapse. These feelings are combined with prepsychotic experiences, for example, anomalies in perception and high suspicion. The combination of these experiences does not necessarily mean a relapse but indicates its high risk.

During CBT, patients are taught to recognize triggers that may lead to the return of psychosis: general anxiety, problems with sleep, mood regulation, and determine their distress factors. At the same time for many patients, the experience of psychosis itself is traumatic and causes postpsychotic disorders. Therefore, it is essential to return the patient with a sense of control, to provide an understanding that the relapse is not catastrophic and there are opportunities to overcome it (Hagen et al 2010).

A randomized controlled trial of the effectiveness of CBT Jackson et al (2008) used the Active Cognitive Therapy for Early Psychosis (ACE) model (Jackson \& McGorry 2008). Therapy included building of the formulation, selecting the list of current problems, learning strategies for coping positive symptoms, treatment of comorbidities, negative symptoms, relapse prevention. In the CBT group was a higher improvement in the social activities of participants but was no effect on positive and negative symptoms. Besides, CBT compared to the group of Befriending therapy by Sensky et al (2000) had no significant difference in the impact of hospitalization. At the same time, the combination of medication plus individual and group psychotherapy (the Relapse Prevention Therapy program) for 12 months courses statistically significantly reduced relapse rates compared to treatment as usual (Gleeson et al 2011). The plan of therapy consisted of five stages, based on the principles of CBT:

1. Engagement in treatment, assessment of the risk of relapse,

2. Building and coordination of formulations focusing on risk factors,

3. Work on reducing risks,

4. The detection of early signs of relapse, the formation of a plan of actions in the event of a recurrence,

5. Additional modules according to the specific risk factors of the participant,

6. Each phase involved specific psychoeducation, motivational interviewing, Socratic questions and behavioral experiments.

Significant indicators for the prevention of psychosis through 12 and 24 months demonstrated in the study Bechdolf et al (2012). The authors compared the group of supportive counseling and an integrated psychological intervention (IPI). IPI included individual cognitivebehavioral therapy (that including psychoeducation, stress management, symptom management), group learning skills (writing activity schedules, social skills, problem-solving), cognitive deficits recovery program (computer programs for training concentration, attention and memory] and family psychoeducation. 


\section{SYSTEMATIC REVIEW}

In contrast to the previous study, the work of Addington et al (2011) on the use of CBT for people at high risk of developing psychosis showed psychosis only in the supportive counseling group. However, the difference was statistically insignificant; both groups had the same reduction in positive symptoms, depression and anxiety. None of the groups showed improvement in social functioning and negative symptoms.

\section{Effect on Comorbid Symptoms and Disorders}

Most articles dedicated to comorbid states and disorders are descriptions of clinical cases, such as depression and anxiety. Post-psychotic depression is a widespread condition and leads to significant complications and worsening of symptoms. The presence of post-psychotic depression is often viewed as an unpleasant prognostic indicator of recovery and reintegration into society.

Only one study in the sample had randomized controlled trial format and included an assessment of the effects on the comorbidities as an additional goal. A follow-up study of patients with high-risk psychosis status by Mark van der Gaag et al (2012) showed improvement in comorbid state at the end of treatment by CBT plus TAU. The evaluation of clinical depression reduced to less than $20 \%$ at the 18 -month follow-up, while clinical social phobia also decreased to less than $20 \%$.

Further studies are descriptions of clinical cases. They indicate the success of cognitive-behavioral interventions when working with patients who have undergone the first psychotic episode and had symptoms of depression or anxiety.

Thus, with the help of CBT significant reductions in depressive and negative symptoms were achieved in the study by Singer et al (2013). Also, participants $(n=10)$ noted a reduction in the feeling of hopelessness and loss, as well as an increase in self-esteem. All participants received treatment with atypical antipsychotic drugs, and half of them received antidepressants.

In 2013, Alison Welfare-Wilson and Rebecca Newman 2013 published a paper on the effectiveness of group therapy for comorbid symptoms patients $(n=7)$ with the first psychotic episode. Group CBT was held once a week in the form of a two-hour session. The therapy lasted 12 weeks and focused on the topics: socialization into the CBT model; identification of co-strategies; mindfulness; relaxation techniques; behavioral experiments; filling in a diary of thoughts (identification of thinking misconceptions, "hot" cognition); recognition of relapse symptoms. The effectiveness of the intervention was evaluated after three months, and the results showed a statistically significant reduction in symptoms of depression $(p=0.06)$, anxiety $(p=0.05)$, general stress $(p=0.014)$.

Monitoring of CBT by two patients with the first psychotic episode by the Sönmez et al (2014) showed improvements in the Global Assessment of Functioning Scale, reduction of depression symptoms, self-esteem and PANSS scores reduction of symptoms. During the six months of the study, participants had 26 sessions of the CBT: creation a list of patient problems, the building of the formulation and explanation of the $A B C$ model. At the same time, Domenichetti \& Pozza (2014) also described the successful experience of cognitivebehavioral therapy in the first psychotic episode of one patient and its effect on the associated symptoms of depression and social anxiety. The treatment consisted of 21 sessions once a week in outpatient settings and consisted of two phases. According to the results of the observation, there were no relapses of psychotic symptoms, reduction of depressive symptoms and symptoms of social anxiety, increased daily activity, number of social contacts, involvement in work and education.

We recognize the limitations associated with the analysis of these articles, namely, that the study did not compare CBT with other interventions, including medical treatment. In our opinion, it limits the present review, but gives prospects for further research, considering the description of the cases and features of the techniques used.

\section{CONCLUSIONS}

The system of early interventions in the primary psychotic episode should be considered as a multidisciplinary set of activities and include pharmacotherapy, interventions aimed to support the family, cognitive-behavioral therapy, social skills training and crisis management. According to the data, CBT-techniques are an effective and scientifically based method for the combined treatment of patients in the early stages of psychotic disorders. CBT early interventions reduce the severity of positive and negative symptoms; improve access to engagement with treatment, which also reduces hospital admissions and relapse rates.

The theory confirms the necessity of all components of early services and points to the importance of open presentation of information to the patient, analytical work on the symptoms and their prevalence, possible causes, and triggers in each particular case. Own effectiveness has proved the technique of useful coping strategies management of psychotic symptoms and skills for social adaptation. Some studies also suggest that standardized CBT protocols for comorbidities such 
as depression or anxiety disorders may be used for patients with psychotic disorders. Useful were both individual and group formats for the CBT.

According to the analyzed studies, there are certain stages, techniques, and programs of cognitivebehavioral therapy that have shown the highest effectiveness in the early stages of the psychotic disorders. However, despite the growth of evidencebased data on the efficacy of the use of CBT in the complex of early intervention, discussion about their preference and specificity is actual. It should be noted that the one limitation of the present review is the lack of randomized controlled trials included in a study. Some trials include CBT early interventions and additional antipsychotic courses in both trial groups and that could impact the effects of early intervention. Also, a small number of early intervention trials provided long-term data, limiting the comparison and review.

Based on this study, recommendations for the use of cognitive-behavioral therapy for the first psychotic episode as a method of early psychotherapeutic intervention can be formulated. The work contains a theoretical overview of the fundamental principles of cognitive-behavioral therapy of psychosis and the results of the study can be used in practice. The present review can be useful for psychiatrists, psychologists, social workers and anyone who works with people at high risk of psychosis, with people who experienced the first psychotic episode or are in the recovery stage after it and need help in relapse prevention.

\section{Contribution of authors}

AO analyzed data, contributed in results interpretation and text writing. SC participated in the planning of the research, data collection and assisted in results interpretation. All authors critically evaluated and approved the paper submission.

\section{Reference:}

Addington J. (2005) Implementing cognitive-behavioural therapy for first-episode psychosis. The British Journal of Psychiatry 48:7276. https://doi.org/10.1192/bjp.187.48.s72

Addington J. (2011) A randomized controlled trial of cognitive behavioral therapy for individuals at clinical high risk of psychosis. Schizophrenia Research 125(1): 54-61.

Addington J., Marshall C. \& French P. (2012) Cognitive Behavioral Therapy in Prodromal Psychosis. Current Pharmaceutical Design 18(4).

Bechdolf A., Wagner M., Ruhrmann S., et al. (2012) Preventing progression to first-episode psychosis in early initial prodromal states. The British Journal of Psychiatry 200(1):22-29. https://doi. org/10.1192/bjp.bp.109.066357.

Bechdolf A., Müller H. \& Stützer H. (2017) A Randomized Controlled Trial for the Prevention of First-Episode Psychosis Comparing Cognitive-Behavior Therapy (CBT), Clinical Management, and Aripiprazole Combined and Clinical Management and Placebo Combined. Schizophrenia Bulletin 43(1):56-57. https://doi. org/10.1093/schbul/sbx021.146.

Bird V., Premkumar P. \& Kendall T. (2010) Early intervention services, cognitive-behavioural therapy and family intervention in early psychosis: systematic review. The British Journal of Psychiatry 197(5):350-356. https://doi.org/10.1192/bjp.bp.109.074526

Chung Young-Chul, Yoon Kil-Sang, \& Park Tae-Won (2012) Group Cognitive-Behavioral Therapy for Early Psychosis. Cognitive Therapy and Research 37(2): 403-411. https://doi.org/10.1007/ s10608-012-9460-9.

Freeman D., Dunn G., Startup H., Pugh K., Cordwell J., Mander H., Cernis E., Wingham G., Shirvell K., Kingdon D. (2015) Effects of cognitive behaviour therapy for worry on persecutory delusions in patients with psychosis (WIT): a parallel, single-blind, randomised controlled trial with a mediation analysis. Lancet Psychiatry 2(4): 282-283. https://doi.org/10.1016/S2215-0366(15)00039-5.

Gleeson J.F.M. \& McGorry P.D. (2003) Psychological interventions in early psychosis a treatment handbook. - 308c. - (Wiley)

Gleeson J.F.M., Cotton S.M. \& Alvarez-Jimenez M. (2011) A Randomized Controlled Trial of Relapse Prevention Therapy for First-Episode Psychosis Patients: Outcome at 30-Month Follow Up. Schizophrenia Bulletin 39(2): 436-448. https://doi.org/10.1093/ schbul/sbr165

Hagen R., Turkington D., \& Berge T. (2010) CBT for Psychosis: A Symptom-based Approach. - London, 2010. - 296 c. - (Routledge).

Jackson H.J. \& McGorry P.D. (2008) Acute-phase and 1-year followup results of a randomized controlled trial of CBT versus Befriending for first-episode psychosis: the ACE project. Psychological Medicine 38(5):725-735. https://doi.org/10.1017/ S0033291707002061.

Jones C., Hacker D., Cormac I., Meaden A., \& Irving C.B. (2012) Cognitive behaviour therapy versus other psychosocial treatments for schizophrenia. Cochrane Database of Systematic Reviews, 4. https://doi.org/10.1002/14651858.CD008712.pub2.

Marshall M. \& Rathbone J. (2011) Early Intervention for psychosis. Cochrane Database of Systematic Reviews, 6. https://doi. org/10.1002/14651858.CD004718.pub3.

Mehl S. \& Werner D. (2015) Does Cognitive Behavior Therapy for psychosis (CBTp) show a sustainable effect on delusions? A meta-analysis. Frontiers in Psychology, 6. https://doi.org/10.3389/ fpsyg.2015.01450.

Ministry of Health of Ukraine (2007) Notice on the publication of a draft order of the Ministry of Health of Ukraine No59. "Clinical protocol of medical care for the patient with first psychosis". Retrieved from: http://medstandart.net/browse/2305.

Ministry of Health of Ukraine (2016) Notice on the publication of a draft order of the Ministry of Health of Ukraine "On approval and implementation of medical technology documents on the standardization of medical care for schizophrenia". Retrieved from: http://old.moz.gov.ua/ua/print/dn_20160702_0.html.

Morrison A.P., French P. \& Stewart S.L.K. (2012) Early detection and 


\section{SYSTEMATIC REVIEW}

intervention evaluation for people at risk of psychosis: multisite randomised controlled trial. BMJ 344. https://doi.org/10.1136/ bmj.e2233.

Morrison A.P., Law H. \& Carter L. (2018) Antipsychotic drugs versus cognitive behavioural therapy versus a combination of both in people with psychosis: a randomised controlled pilot and feasibility study. Lancet Psychiatry 5(5):381-383. http://dx.doi. org/10.1016/S2215-0366(18)30123-8.

Morrison N.K. (2009) Cognitive Behavior Therapy for People with Schizophrenia. Psychiatry (Edgemont) 6(12):32-39. https://www. ncbi.nlm.nih.gov/pmc/articles/PMC2811142/.

National Institute for Health and Care Excellence (2014) Core Interventions in the Treatment and Management of Schizophrenia in Adults in Primary and Secondary Care. Retrieved from: https:// www.ncbi.nlm.nih.gov/books/NBK11681/.

National Institute of Mental Health (2015) Fact Sheet: First Episode Psychosis. Retrieved from:https://www.nimh.nih.gov/health/ topics/schizophrenia/raise/fact-sheet-first-episode-psychosis. shtml

Nowak I. \& Sabariego C. (2016) Disability and recovery in schizophrenia: a systematic review of cognitive behavioral therapy interventions. BMC Psychiatry 16. https://doi.org/10.1186/s12888-016-0912-8.
Singer A.R., Addington D.E. \& Dobson K.S. (2014) A Pilot Study of Cognitive Behavior Therapy for Depression in Early Psychosis. Cognitive and Behavioral Practice 21(3): 323-334. https://doi. org/10.1016/j.cbpra.2013.08.004.

Sönmez N., Hagen R. \& Andreassen O.A. (2014) Cognitive Behavior Therapy in First-Episode Psychosis with a Focus on Depression, Anxiety, and Self-Esteem. Cognitive and Behavioral Practice 21(1):43-54. https://doi.org/10.1016/j.cbpra.2013.06.001

Turner D.T. \& van der Gaag M. (2014) Psychological interventions for psychosis: a meta-analysis of comparative outcome studies. The American Journal of Psychiatry 171(5):523-538. https://doi. org/10.1176/appi.ajp.2013.13081159.

Van der Gaag M., Nieman D.H. \& Rietdijk J. (2012) Cognitive Behavioral Therapy for Subjects at Ultrahigh Risk for Developing Psychosis: A Randomized Controlled Clinical Trial. Schizophrenia Bulletin 38(6):1180-1188. https://doi.org/10.1093/schbul/sbs105.

Welfare-Wilson A. \& Newman R. (2013) Cognitive behavioural therapy for psychosis and anxiety. British Journal of Nursing 22(18). https://doi.org/10.12968/bjon.2013.22.18.1061.

World Health Organization (2012) Psychoeducation, family interventions and cognitive-behavioural therapy. Retrieved from: http://www. who.int/mental_health/mhgap/evidence/psychosis/q10/en/. 